\title{
Semiparametric Estimation of Multi-Asset Portfolio Tail Risk
}

\author{
Alexandra Dias* \\ School of Management \\ University of Leicester \\ Final draft \\ Journal of Banking and Finance, 2014 \\ DOI: 10.1016/j.jbankfin.2014.05.033
}

\begin{abstract}
When correlations between assets turn positive, multi-asset portfolios can become riskier than single assets. This article presents the estimation of tail risk at very high quantiles using a semiparametric estimator which is particularly suitable for portfolios with a large number of assets. The estimator captures simultaneously the information contained in each individual asset return that composes the portfolio, and the interrelation between assets. Noticeably, the accuracy of the estimates does not deteriorate when the number of assets in the portfolio increases. The implementation is as easy for a large number of assets as it is for a small number. We estimate the probability distribution of large losses for the American stock market considering portfolios with ten, fifty and one hundred assets of stocks with different market capitalization. In either case, the approximation for the portfolio tail risk is very accurate. We compare our results with well known benchmark models.
\end{abstract}

JEL Classification: C51, G01, G11, G17.

KEY WorDs: Multi-asset portfolios, risk management, tail probability, tail risk, multivariate extreme value theory, Value-at-Risk.

${ }^{*}$ Correspondence address: Alexandra Dias, School of Management, University of Leicester, LE1 7RH Leicester, UK. E-mail: Alexandra.Dias@le.ac.uk. 


\section{Introduction}

Multi-asset managers hedge risk by investing in assets that ideally have low correlations. Unfortunately through markets integration and financial crises, correlations between assets have become more often positive and stronger. Expressions like "no place to hide" are now commonplace in the finance and investments news. In such environment it is paramount for the multi-asset manager to accurately monitor the tail risk of his portfolio.

The risk of incurring very large losses in a financial position is commonly measured by Value-at-Risk (VaR) or Expected Shortfall (ES). The computation of tail risk measures boils down to the estimation of the probability of observing very large losses. The main challenge presented by this task comes from the fact that large losses are by their own nature scarce. With few observations it is not trivial to implement successfully statistical methods.

Classical finance theory assumes that asset returns have a multivariate normal distribution. Yet, the normality assumption leads often to the underestimation of the probability of large losses. As a natural extension, the Student- $t$ distribution allows for heavy-tails which help to overcome the "optimism" of the normal model relating the frequency of large losses. The drawback of the Student- $t$ model is that financial returns are typically asymmetric due to many small gains and few large losses. The Student- $t$ model is symmetric, hence assuming that the distribution of losses and gains have the same shape is often unreasonable. Another well known model is a mixture of normal distributions. We include a mixture of normal distributions as a benchmark model in our study.

In fact, to estimate tail risk we only need a good model for the (long) tail of the distribution of the losses. Extreme Value Theory (EVT) models focus on the tail of the distribution only. Hence, EVT provides a tailor made methodology to estimate the probability of large losses.

Multi-asset portfolios exist because managers want to take advantage of the interaction between assets. This is important from the modeling point of view. Portfolio losses are the result not only of the individual assets performance but also, and very importantly, from the 
interaction between assets. This raises the question of the choice between univariate and multivariate models for the tail risk. Multivariate models make use of information from individual assets while univariate models use the information already synthesized in the portfolio returns. Hence, from the accuracy point of view ideally we would prefer the multivariate approach.

Multivariate EVT models exist. These are parametric models where a dependence structure for the large losses of several assets is imposed. However, since large losses are rare, parameter estimation and specification tests become a serious challenge. Our solution is to use a semiparametric EVT estimator for computing portfolio tail risk. This estimator captures the statistical tail features of each individual asset as well as their interaction, without imposing a specific dependence structure. In addition it can be used with high-dimensional portfolios.

The probability of multi-asset portfolio losses is used in several contexts by managers and analysts as in portfolio selection with downside risk, and in particular for estimating VaR. The advent of modern finance crystalized the need of modeling large losses in multi-asset positions. The safety-first principle of Roy (1952) constrains portfolio selection on a small probability of large losses. Portfolio selection with downside risk constraints and risk management practice have often used VaR as the risk measure for large losses. Traditionally, these probabilities and risk measures have been computed based on the assumption that asset returns have a multivariate normal distribution. Indeed, if asset returns are assumed to be multivariate normal then closed form solutions can be used for multi-asset portfolio VaR and the calculation of constrained portfolio weights; see for instance Alexander and Baptista (2002) and Alexander and Baptista (2008). However, risk management with VaR constraints (VaR-RM) leads one to select positions with higher variance. Furthermore, as Basak and Shapiro (2001) show, when losses larger than VaR occur they are larger if VaR-RM is used than if VaR-RM is not used. All these findings argue for good models and estimators for the tail of the loss distribution.

Assuming a multivariate normal distribution for asset price returns implies that, asymptotically, large losses of individual assets occur independently. In contrast, especially in periods of 
crisis, we observe that several financial assets can suffer simultaneously dramatic losses. The fact that financial data often does not support the hypothesis of a normal distribution for asset returns suggests the use of a more appropriate model. The problem with using an alternative approach is that closed forms for VaR or ES are not known for most probability distributions. One possible alternative is to use Tchebychev's inequality to approximate the tail of a portfolio distribution. But Arzac and Bawa (1977), for instance, found that this inequality produces poor approximations. As a response, Arzac and Bawa (1977) use a nonparametric model for the asset returns distribution. Later, Gourieroux et al. (2000) also model the portfolio returns distribution using a nonparametric model. However, there is no need to model the entire distribution in order to obtain the probability of large losses. It is sufficient to model the tail of the distribution. EVT was developed specifically for modeling large events. Hence, EVT provides a set of tools appropriate to model the probability of portfolio large losses.

EVT has been used in finance mainly with univariate models. Univariate EVT models have been used in different settings by numerous authors. For instance, Jansen et al. (2000), Longin (2000), and McNeil and Frey (2000) compute portfolio VaR by modeling the portfolio returns with univariate EVT. Susmel (2001) implements portfolio selection with safety first using univariate EVT to model the portfolio large losses. Zhang and Shinki (2007) use univariate EVT to model high frequency asset returns. Brooks et al. (2005) use univariate EVT models and compares them with other approaches to model large return losses.

Although the use of univariate EVT models can result in an important loss of information, multivariate EVT models are far less used in finance. Hyung and de Vries (2007) reduce the multivariate problem to the univariate case by modeling the asset returns with a one factor model and modeling the (independent) idiosyncratic component of the tail risk with an EVT model. Longin (2000) computes the VaR of multivariate positions using a decomposition into a set of risk factors. He models the extreme dependence of risk factors as a linear combination of the dependence function of total dependence and asymptotic dependence. Daul et al. (2003) 
use the grouped $t$-copula to capture the risk in a large set of risk factors in an application to credit risk. Other authors, as Ang and Chen (2002), capture the multivariate structure by modeling correlations with regime switching models. Specifically, Tsafack and Garcia (2011) use an EVT copula in a regime-switching copula model to capture the joint extreme behavior of asset returns. Multivariate EVT models are also implemented by Longin and Solnik (2001) and by Poon et al. (2004). In these articles the logistic function is used to model the dependence of extreme asset returns. These models are completely parametric and the estimation of their parameters is usually difficult due to the lack of historical (extreme) observations.

In this article we estimate the tail risk of multi-asset portfolios using a semiparametric EVT approach. We use an estimator which assumes a parametric EVT specification for the univariate asset tail risk combined with a nonparametric estimator for the dependence structure of the portfolio large losses. This reduces the problem of model misspecification, increasing the accuracy of the results compared with fully parametric models. Another advantage of the methodology is that the estimation of the portfolio tail risk does not become more difficult as the number of assets in the portfolio increases.

To the best of our knowledge this method has not been used before in tail risk estimation. The contribution of this article has two aspects. The estimation of the tail of the distribution of the portfolio large losses, and the specific estimation of the portfolio tail risk using VaR as a measure of risk.

We estimate the tail of three equity portfolios. The first portfolio has ten assets, the second portfolio has fifty assets and the third portfolio has one hundred assets. We compare the performance of the semiparametric estimator with the performances of a mixture of normal distributions, and of a Student- $t$ model.

For the three portfolios described above we estimate tail risk with VaR computed at several levels of confidence. The accuracy of the tail risk estimates is inspected using a backtesting procedure. 
This paper is organized as follows. Section 2 summarises the theoretical foundation of the semiparametric estimator for tail risk. Section 3 describes the statistical estimation methodology. In Section 4 we estimate the distribution of large losses of the three equity portfolios, and the portfolios tail risk. Section 5 concludes this article.

\section{The semiparametric estimator for portfolio large losses}

Very large movements of asset prices, although often implying financial distress, do not occur frequently. Modeling rare losses is a challenging task from the statistical point of view. The natural tool to quantifying risk in positions involving assets subject to rare events is EVT. Here we focus on quantifying risk of portfolios with possibly a large number of assets.

EVT provides a model for the multivariate distribution of the maximum return ${ }^{1}$ observed for each asset over a period of time. We denote by $\mathbf{R}=\left(R_{1}, R_{2}, \ldots, R_{d}\right)$ the random vector representing the one period loss returns of $d$ assets. A main result in extreme value theory is that for the distributions commonly used for financial returns, there is a limit distribution for the componentwise maxima. In the following we summarize this result and show how to use consequent results in quantifying multi-asset portfolio large losses.

Let $\mathbf{R}_{t}, t=1,2, \ldots, n$ denote the vector of $d$ asset returns in period $t$. If the random vectors $\mathbf{R}_{1}, \mathbf{R}_{2}, \ldots, \mathbf{R}_{n}$ are independent and identically distributed, drawn from a distribution $F_{\mathbf{R}}$, then the distribution of the componentwise maxima is the power $F_{\mathbf{R}}^{n}(\mathbf{x})$. This distribution is usually unknown. To overcome this problem, extreme value theory derives the limit distribution of $F_{\mathbf{R}}^{n}(\mathbf{x})$ when $n$ goes to infinity, resembling the approach used for deriving the Central Limit Theorem (CLT). Like with the CLT case, the componentwise maxima have to be standardized in order to have a non-degenerate limit distribution. We denote by $\tilde{\mathbf{R}}_{n}$ the vector of standardized

\footnotetext{
${ }^{1}$ Often we are interested in modeling the minimum return but this is equivalent to modeling the maximum loss. Hence the results presented for the maximum can be used for the minimum return.
} 
componentwise maxima, so that,

$$
\tilde{\mathbf{R}}_{n}=\mathbf{a}^{-1}\left(\max \left(\mathbf{R}_{1}, \mathbf{R}_{2}, \ldots, \mathbf{R}_{n}\right)-\mathbf{b}\right)
$$

where $\mathbf{a}$ and $\mathbf{b}$ are vectors of scale and location coefficients depending on $n .^{2}$

From extreme value theory (see de Haan and Resnick (1977)) there is a non-degenerate limit distribution $G(\mathbf{x})$ for the normalized maxima, the so called extreme value distribution, such that

$$
\lim _{n \rightarrow \infty} P\left(\tilde{\mathbf{R}}_{n} \leq \mathbf{x}\right)=G(\mathbf{x})
$$

This implies convergence for the marginal distributions, in particular, that each univariate marginal $G_{j}(x), j=1, \ldots, d$ has an extreme value distribution. In fact, there are normalizing constants $a_{j}>0$ and $b_{j} \in \mathbb{R}$ such that each univariate marginal distribution is of the form

$$
G_{j}(x)=\exp \left(-\left(1+\gamma_{j} \frac{x-b_{j}}{a_{j}}\right)^{-1 / \gamma_{j}}\right),
$$

for $1+\gamma_{j}\left(x-b_{j}\right) / a_{j}>0$ and where $\gamma_{j} \in \mathbb{R}$ is the shape parameter.

Let $G$ be a univariate extreme value distribution. If $\gamma<0$ then $G$ is a Weibull distribution, if $\gamma=0$ then $G$ is a Gumbel distribution and if $\gamma>0$ then $G$ is a (heavy tailed) Fréchet distribution (see Gnedenko (1943)). In the last case where $\gamma>0$ the shape parameter $\gamma$ is known as the tail index.

Any multivariate parametric approach to estimate the probability of a loss requires a model for the distribution of the vector of returns $\mathbf{R}$. A multivariate model for $\mathbf{R}$ allows us to estimate the probability of events which are a function $f(\mathbf{R})$ of these returns. In our case, we want to estimate the probability of a portfolio large loss. Hence, the function $f: \mathbb{R}^{d} \rightarrow \mathbb{R}$ is the average of the individual asset returns weighted by the proportion of each asset in the portfolio. We want to estimate the probability that a portfolio loss is larger than a given target threshold $L$. This means, in terms of our notation, that we want to estimate $P(f(\mathbf{R})>L)$.

\footnotetext{
${ }^{2}$ Here and elsewhere in this article, operations (maximum, addition, multiplication and taking powers) are componentwise.
} 
There are several possible scenarios (in terms of individual asset returns) for which the portfolio will suffer a loss larger than the given target. We denote by $C$ this set of possible multivariate returns, $C \subseteq \mathbb{R}^{d}$, implying a portfolio loss larger than $L$. This means that estimating the probability $P(f(\mathbf{R})>L)$ is equivalent to estimating $P(\mathbf{R} \in C)$ where $C$ contains all the possible joint returns $\mathbf{R}$ such that $f(\mathbf{R})>L, C=\left\{\mathbf{R} \in \mathbb{R}^{d}: f(\mathbf{R})>L\right\}$.

Given its nature, of being rare, usually there are few observations of portfolio large losses in the past returns. As a consequence often few observations fall in the set $C$. This fact prevents the use of statistical estimation methodologies whose performance relies on the existence of a considerable number of observations. The following result from extreme value theory is key for overcoming this problem.

If $G$ is an extreme value distribution as in (2) then (see de Haan and Resnick (1977)) there exists a finite measure $\nu$ such that, for any Borel set $A$ of $[0, \infty]^{d} \backslash \mathbf{0}$ and for a scaling constant $c>0$,

$$
c \nu(c A)=\nu(A)
$$

provided that $A$ is bounded away from the origin. The measure $\nu$ is called the exponent measure. The scaling property of the exponent measure, given by (4), is very useful to estimate the probability of observing a portfolio large loss. We first explain the main intuition and then we describe the method in detail.

The set $C$, of which we want to estimate the probability, typically is not close to the centre of the returns distribution and rather far in the tail. But if we divide every point in $C$ by a constant (appropriately determined as explained in the following), we obtain another set which is much closer to the centre of mass of the distribution where many observations exist. As a consequence we can estimate the probability of this set which is closer to the centre of the support of the distribution using standard statistical methods. Finally, given the scaling property in equation (4), we know that the probability of the set far in the tail is given by the probability of the set closer to the centre of the distribution divided by the constant $c$. The 
method is explained in more detail in the following.

To apply the scaling property (4) we must first rescale the univariate margins using the normalizing constants and the shape parameters defined in equation (3). Accordingly, the original returns $\mathbf{R}$ are transformed into the pseudo-return vectors $\tilde{\mathbf{R}}$,

$$
\tilde{\mathbf{R}}:=\left(1+\gamma \frac{\mathbf{R}-\mathbf{b}}{\mathbf{a}}\right)^{1 / \gamma}
$$

To estimate the probability of events on the space of the pseudo-returns in terms of the measure $\nu$, we use a result from de Haan and Resnick (1977). Let $A$ be any Borel set of $[0, \infty]^{d} \backslash \mathbf{0}$ bounded away from the origin and such that $\nu(\partial A)=0$. For a given $k$ such that $0<k \leq n$, we have that

$$
\lim _{n \rightarrow \infty} \frac{n}{k} P(\tilde{\mathbf{R}} \in A)=\nu(A)
$$

as $n \rightarrow \infty, k \rightarrow \infty$ and $k / n \rightarrow 0$. The probability $P(\tilde{\mathbf{R}} \in A)$ above depends on $n$ because $\tilde{\mathbf{R}}$ uses $\mathbf{a}$ and $\mathbf{b}$ which depend on $n$ by (1). $k$ is the number of observations, depending upon $n$, which are large enough to be considered in the tail of the distribution. The question of how to choose $k$ for a given sample is addressed in the next section within the estimation methodology.

Using (6), the probability of having joint returns in a set $C$ implying losses larger than a target large loss $L$, can be obtained by writing $C$ as a transformation of a measurable set $A \subseteq[0, \infty]^{d} \backslash \mathbf{0}$ as

$$
C=\mathbf{a} \frac{(c A)^{\gamma}-\mathbf{1}}{\gamma}+\mathbf{b} \quad \text { or } \quad c A=\left(\mathbf{1}+\gamma \frac{C-\mathbf{b}}{\mathbf{a}}\right)^{1 / \gamma}
$$

where $c$ is a positive scaling constant as in (4).

As noted before, as the set $C$ corresponds to very large portfolio losses there are typically very few, if any, observations in this set. This scarcity of observations in $C$ translates into the same lack of observations in its transformed set $c A$ which invalidates the use of an empirical estimator for $\nu(c A)$. But the normalizing transformation (5) shifts the return vectors towards the point $\mathbf{1}$. Hence, if we impose the requirement that the point $\mathbf{1}$ belongs to the boundary of 
$A$ then the set $A$ will have enough observations to allow the estimation of $\nu(A)$. This defines the constant $c$.

Finally, given that $\mathbf{1}$ is on the boundary of $A$, we have that the probability of having an observation in the set $C$, and consequently a portfolio large loss, is

$$
\begin{array}{rlrl}
p & :=P(\mathbf{R} \in C) & \\
& =P\left(\mathbf{R} \in \mathbf{a} \frac{(c A)^{\gamma}-\mathbf{1}}{\gamma}+\mathbf{b}\right) \\
& =P(\tilde{\mathbf{R}} \in c A) & \\
& \approx \frac{k}{n} \nu(c A) & \text { as } n \rightarrow+\infty & {[\text { by }(6)]} \\
& =\frac{k}{n c} \nu(A) & {[\text { by }(4)] .}
\end{array}
$$

The scaling property (4) is the key for the estimation of the probability of portfolio losses possibly never observed before. Result (8) shows how to obtain the probability of rare events depending on the returns of several assets.

\section{Portfolio large losses: estimation methodology}

In order to use expression (8) to estimate the probability of a portfolio large loss originated by a realization of a joint asset return in $C$, we need to obtain estimates for $c, k, A$ and $\nu(\cdot)$ (the value of $n$ is the sample size). Estimation methods for these unknowns are addressed in this section.

To perform the transformation of the margins of the individual asset returns we need to estimate the shape parameter $\gamma$, and the location and scale parameters, $a$ and $b$ respectively. Consider the random sample of size $n$ of univariate asset returns, $R_{1}, R_{2}, \ldots, R_{n}$. The order statistics are defined by the ordered sample returns denoted as

$$
R_{(n)} \leq R_{(n-1)} \leq \ldots \leq R_{(1)}
$$

The random variable $R_{(k)}$ is called the $k$ th upper order statistic. 


\subsection{The shape parameter (tail index if $\gamma>0$ )}

Define the function,

$$
M_{r}(R):=\frac{1}{k} \sum_{i=1}^{k}\left(\log R_{(i)}-\log R_{(k+1)}\right)^{r} .
$$

for $r=1,2$. The so called moment estimator of the shape parameter $\gamma($ Dekkers et al. (1989)), is given by

$$
\hat{\gamma}:=M_{1}(R)+1-\frac{1}{2}\left(1-\frac{M_{1}(R)^{2}}{M_{2}(R)}\right)^{-1} .
$$

This is a consistent estimator of the shape parameter. Under an additional technical condition (see Dekkers et al. (1989)) we have that if $\gamma \geq 0$ then $\sqrt{k}(\hat{\gamma}-\gamma)$ has asymptotically a normal distribution with mean zero and variance $1+\gamma^{2}$. For the case $\gamma<0$ the distribution of the statistic $\hat{\gamma}$ is also known and can be found in Dekkers et al. (1989)). In our empirical study we only need the distribution of $\hat{\gamma}$ for $\gamma \geq 0$. All the estimates of the shape parameter are significantly larger than zero.

We use the moment estimator for the shape parameter because we do not know beforehand if all portfolio components have a heavy-tail. The moment estimator is appropriate in this case because it can be used whether $\gamma>0$ or $^{3} \gamma \leq 0$.

\subsection{The normalizing constants}

To estimate the normalizing constants $a$ and $b$, the parameters of the univariate extreme value distribution given in (3), we use estimators studied by Dekkers et al. (1989). Define the functions,

$$
\bar{t}=t \wedge 0, \quad \rho_{1}(t)=\frac{1}{1-\bar{t}} \quad \text { and } \quad \rho_{2}(t)=\frac{2}{(1-\bar{t})(1-2 \bar{t})}
$$

then estimators for $a$ and $b$ are

$$
\hat{b}:=R_{(k+1)} \quad \text { and } \quad \hat{a}:=\frac{R_{(k+1)} \sqrt{3 M_{1}(R)^{2}-M_{2}(R)}}{\sqrt{3\left(\rho_{1}(\hat{\gamma})\right)^{2}-\rho_{2}(\hat{\gamma})}}
$$

respectively.

\footnotetext{
${ }^{3}$ This is not possible with the Hill estimator (Hill (1975)) which can only be used when $\gamma>0$.
} 


\subsection{The exponent measure}

In order to estimate the exponent measure $\nu(\cdot)$ first we transform the multivariate returns $\mathbf{R}_{1}, \mathbf{R}_{2}, \ldots, \mathbf{R}_{n}$ according to (5). However, as the true values of the normalizing constants and of the shape parameter are not known we have to use their estimators. Consequently, we obtain the estimates of the normalized returns, which we denote by $\hat{\mathbf{R}}_{1}, \hat{\mathbf{R}}_{2}, \ldots, \hat{\mathbf{R}}_{n}$, as

$$
\hat{\mathbf{R}}_{t}:=\left(\mathbf{1}+\hat{\gamma} \frac{\mathbf{R}_{t}-\hat{\mathbf{b}}}{\hat{\mathbf{a}}}\right)^{1 / \hat{\gamma}}
$$

for $t=1,2, \ldots, n$.

We estimate the exponent measure $\nu(\cdot)$ using the nonparametric estimator suggested by de Haan and Resnick (1993),

$$
\hat{\nu}_{n}(A):=\frac{1}{k} \sum_{t=1}^{n} \mathbb{I}\left(\hat{\mathbf{R}}_{t} \in A\right)
$$

where $\mathbb{I}$ denotes the indicator function. Recall that the tail of the distribution of each individual asset is assumed to have a parametric distribution given by (3). However, the estimation of the extremal dependence structure using (12) does not assume any particular parametric form. It becomes clear at this point why this approach to the estimation of portfolio large losses is semiparametric. We do not have to assume any parametric model for the dependence structure of large losses. The fact that the semiparametric estimator does not suffer from the curse of dimensionality is also clear now from equation (12). It is as easy to implement equation (12) in low dimension as in high dimension.

\subsection{The scaling constant $c$ and the set $A$}

To estimate the probability of a portfolio large loss using (8) we need to estimate the scaling constant $c$ and the set $A$. We have to impose a condition in order to have $c$ and $A$ uniquely defined. We want $A$ to be such that we can use the nonparametric estimator $\hat{\nu}(\cdot)$. Hence, $A$ should contain pseudo-observations $\hat{\mathbf{R}}$. That happens if we impose the requirement that the 
point $\mathbf{1}$ is on the boundary of $A$ (Dekkers et al. (1989)), given that $\hat{\mathbf{R}}$ are obtained using the standardization (11).

Recall that we define the set $C$, for some target portfolio large loss $L$, using the function $f(\cdot)$ such that

$$
C=\{\mathbf{R} \mid f(\mathbf{R}) \geq L\}
$$

Each point $\mathbf{R}$ in the set $C$ can be written as the transform of a point $\hat{\mathbf{R}}$ by the inverse mapping of (11),

$$
\mathbf{R}=\mathbf{a} \frac{\hat{\mathbf{R}}^{\gamma}-\mathbf{1}}{\gamma}+\mathbf{b}
$$

At this point we assume that the function $f$ is defined for the case when all returns take the same value. This assumption should not be restrictive in practice. Hence, there exists a value $x$ such that $\mathbf{R}=(x, x, \ldots, x)$ is solution of the equation $f(\mathbf{R})=L$ which is equivalent to the existence of a value $c$ such that $c .1$ is a solution of the equation

$$
f\left(\mathbf{a} \frac{(c \mathbf{1})^{\gamma}-\mathbf{1}}{\gamma}+\mathbf{b}\right)=L
$$

If $c$ is the solution of this equation, then the point $\mathbf{1}$ is on the boundary of $A$. Since we have only estimates of $\mathbf{a}, \mathbf{b}$ and $\gamma$ we actually obtain an estimate $\hat{c}$ of $c$ as solution of (13).

Finally, from (7) we define

$$
\hat{A}:=\frac{1}{\hat{c}}\left(\mathbf{1}+\hat{\gamma} \frac{C-\hat{\mathbf{b}}}{\hat{\mathbf{a}}}\right)^{1 / \hat{\gamma}}
$$

and

$$
\hat{p}:=\frac{k}{n \hat{c}} \hat{\nu}_{n}(\hat{A})
$$

is the estimator of $P(\mathbf{R} \in C)$, the probability of a portfolio loss larger than $L$.

\subsection{The number of upper order statistics $k$}

The choice of the number of upper order statistics $k$ used in the analysis is important. If $k$ is small, which means that we use few upper order statistics, then the parameter estimates 
obtained have a large variance. If we use many upper order statistics, $k$ is large, then the parameter estimates are biased. In our empirical study we set the number of upper order statistics equal to $15 \%$ of the sample size. This is a common value used in the literature; for a recent application see Chavez-Demoulin et al. (2011) and references therein.

\section{Equity portfolio tail risk}

We study the performance of the semiparametric estimator in quantifying the tail risk of multiasset portfolios using three equity portfolios having ten, fifty and one hundred components respectively. The data are prices of individual assets from the S\&P 500, S\&P 400 and S\&P 600 stock indices, obtained from Datastream. The list of assets used can be found in the Appendix in Tables 4 and 5. The portfolios we use are composed of stocks from the three indices in equal proportion. We use data from these three indices in order to include in our study stocks covering a broad range of market capitalization. All the portfolios are equally weighted.

Multi-asset portfolios can be composed of different types of assets as equity, bonds or cash. We choose to use stocks with different market capitalization in our study because it is known that firms with smaller market capitalization typically have higher returns than firms with larger market capitalization (see Banz (1981) for seminal results). Hence, estimating VaR of portfolios composed by stocks with different market capitalization is a relevant application of the semiparametric estimator. The fact that these indices are representative of the market give our results relevance and reliability. Additionally, this is standardized widely available data, hence our results can be easily replicated.

The data covers the period from December 31, 1999 to January 1, 2014. We estimate the tail of the distribution of the portfolio losses using the semiparametric estimator, and compare it with a Student- $t$ and a mixture of four normal distributions. A review of the relevant properties and results on using a mixture of normal distributions to model the unconditional distribution of asset returns can be found in Haas et al. (2004) and references therein. We compute the 
VaR for the ten, fifty and one hundred component portfolios, at the levels of $99 \%, 97.5 \%, 95 \%$ and $90 \%$.

\subsection{Empirical results}

Table 1 presents summary statistics of the daily logarithmic returns for each portfolio, and for subportfolios of assets from the S\&P 600, S\&P 400 and S\&P 500. We refer to the subportfolios of assets from the S\&P 600, S\&P 400 and S\&P 500 as small cap, mid cap, and large cap respectively. The average return is close to zero and tends to decrease with market capitalization. All portfolio components reveal asymmetry having negative sample skewness. The excess kurtosis is very large consistently across all market capitalization components with a tendency to increase with market capitalization. These two statistics suggest that normality would not be a reasonable assumption for the distribution of the tail of the returns distribution. As it is usual in financial returns, the minimum (losses) is often larger than the maximum in absolute value.

Table 1: Summary statistics of the returns on the different portfolios.

\begin{tabular}{lccrrrr} 
Portfolio & Mean & Std. Dev. & Skewness & Kurtosis & Min & Max \\
\hline 10 asset portfolio & & & & & & \\
$\quad$ Small cap & 0.0234 & 2.0948 & -0.1822 & 5.7359 & -18.908 & 13.012 \\
Mid cap & 0.0152 & 1.9030 & -0.7643 & 13.5499 & -23.109 & 16.694 \\
Large cap & 0.0046 & 1.6337 & -0.3239 & 7.7370 & -12.270 & 11.765 \\
Portfolio & 0.0134 & 1.5963 & -0.4488 & 7.6811 & -13.320 & 10.120 \\
& & & & & & \\
50 asset portfolio & & & & & & \\
Small cap & 0.0257 & 1.6491 & -0.2074 & 4.9747 & -12.810 & 9.001 \\
Mid cap & 0.0321 & 1.3239 & -0.3017 & 7.5197 & -11.048 & 10.362 \\
Large cap & 0.0301 & 1.3730 & -0.3247 & 9.8304 & -12.247 & 13.209 \\
Portfolio & 0.0293 & 1.3699 & -0.3443 & 7.9114 & -11.603 & 10.867 \\
100 asset portfolio & & & & & & \\
Small cap & 0.0227 & 1.6435 & -0.2951 & 5.6308 & -13.802 & 9.242 \\
Mid cap & 0.0214 & 1.4123 & -0.4637 & 8.1416 & -12.359 & 10.664 \\
Large cap & 0.0217 & 1.2798 & -0.2803 & 9.0186 & -10.722 & 11.591 \\
Portfolio & 0.0219 & 1.3925 & -0.4200 & 7.6130 & -11.854 & 10.061 \\
\hline
\end{tabular}

Notes: Summary statistics of the daily returns on the ten, fifty and one hundred asset portfolios from small to large market capitalization stocks. The data covers the period from December 31, 1999 to January 1, 2014. In general, returns on low market capitalization stocks have higher mean, and returns on larger market capitalization stocks have higher kurtosis. 
Table 2: Tail index of losses on different market capitalization portfolios.

\begin{tabular}{lcccc} 
& Low cap & Mid cap & Large cap & Portfolio \\
\hline $\begin{array}{l}\text { 10 asset portfolio } \\
\text { Tail Index }\end{array}$ & 0.1679 & 0.2760 & 0.2379 & \\
s.e. & $(0.0374)$ & $(0.0382)$ & $(0.0379)$ & 0.2632 \\
& & & \\
50 asset portfolio & 0.1687 & 0.2296 & 0.2250 & \\
Tail Index & $(0.0373)$ & $(0.0378)$ & $(0.0378)$ & 0.2114 \\
s.e. & & & & $(0.0377)$ \\
$\begin{array}{l}\text { 100 asset portfolio } \\
\text { Tail Index }\end{array}$ & 0.1959 & 0.2455 & 0.2509 & 0.2473 \\
s.e. & $(0.0375)$ & $(0.0379)$ & $(0.0380)$ & $(0.0379)$ \\
\hline
\end{tabular}

Notes: Tail index estimates, $\hat{\gamma}$, for the daily return losses on the ten, fifty and one hundred asset portfolios from small to large market capitalization stocks. The corresponding estimated standard errors are in parentheses. The data covers the period from December 31, 1999 to January 1, 2014.

The semiparametric estimator relies on the assumption that the multivariate limit distribution of the portfolio returns is an extreme value distribution with heavy tailed margins or equivalently that each margin has a positive shape parameter. At first this might seem a restrictive assumption for using the semiparametric estimator. We estimate the shape parameter for the losses of the components of the three portfolios. The results are in Tables 2, 4, and 5 . Tables 4and 5 list the estimates for the individual assets and hence are in the Appendix. The results raise no doubt. Whether for the different portfolios considered or for the individual assets, the shape parameter estimates are always significantly positive. Equity returns have heavy tails across all sizes of market capitalization.

It is interesting to note that although the estimates for the kurtosis tend to increase with market capitalization (see Table 1), the estimates of the tail index for losses do not reveal a definite trend. This seems to be an interesting observation if we associate the tail index of losses with risk. On the one hand stocks with small market capitalization typically have higher returns. On the other hand, they do not reveal higher tail risk. It might be interesting to pursue research on this relation between return and tail risk. 


\subsection{Probability of portfolio large losses}

We use the estimator given by equation (15) to obtain values for the probability of portfolio large losses for the three portfolios with ten, fifty and one hundred assets. For each portfolio we compute estimates for values of losses above the empirical $90 \%$ quantile. Hence, we estimate the $10 \%$ tail of the returns distribution for each portfolio. The results obtained are plotted (in log$\log$ scale) in Figures 1, 2, and 3. The estimated tail probabilities are plotted together with the (survival) empirical distribution. The plot only shows values above the empirical $90 \%$ quantile. The results are very good. The estimates produced by the semiparametric estimator are very close to the empirical distribution up the highest quantiles. A very important observation is that the estimator perfectly captures the heaviness of the tail of the returns distribution by slightly overestimating the probability of large losses. This is in line with the conservative approach necessary in risk management and advocated by the regulators. This excellent result is consistently observed for the portfolio with ten, fifty and one hundred assets. The theoretical fact that the semiparametric estimator does not suffer from the curse of dimensionality is empirically evidenced here with the estimates obtained. The estimates plotted in Figure 3 for the one hundred asset portfolio are not inferior to the estimates obtained for the portfolios with ten and fifty assets (Figures 1 and 2).

In practice, the estimators for tail risk are bounded to be parsimonious and a commonly suggested approach is to estimate tail risk using the univariate portfolio losses avoiding a sometimes unfeasible multivariate modeling. To benchmark our results we estimate the tail probabilities using two univariate models, a mixture of four normal distributions and the Student- $t$ fitted to the univariate portfolio returns. The estimates are plotted in the same Figures 1, 2, and 3. As previously reported in the literature, see Haas et al. (2004) for an overview, the mixture of normal distributions provides a reasonably good approximation to the returns distribution. But given that here we are focussing on the tail of the distribution it is more difficult for the mixture of normals to capture the heaviness of the distribution of large losses. We can see in 


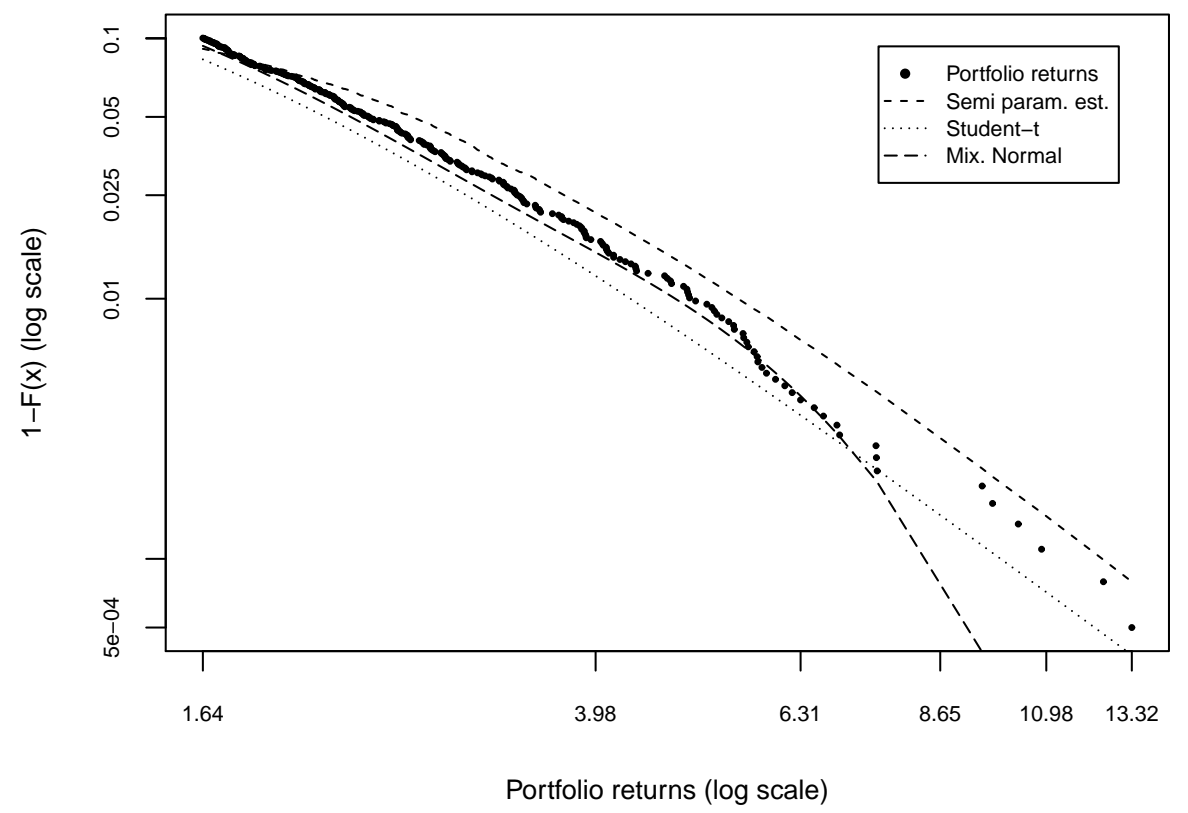

Figure 1: Observed losses (plotted in log-log scale) on the ten asset portfolio together with mixture normal, Student- $t$, and semiparametric approximation. Note that the mixture normal fails to model the tail above very high quantiles.

the figures that the mixture of normal distributions in general underestimates the probability of large losses, most severely for quantiles above 99\%. The Student- $t$ model gives an approximation much better than the mixture of normals above the $99 \%$ quantile but still underestimates the tail probability for all values of losses considered. Note that the difference is read on the vertical axis. The underestimation of tail probabilities, based on empirical past observations, represents a high risk in portfolio management. 


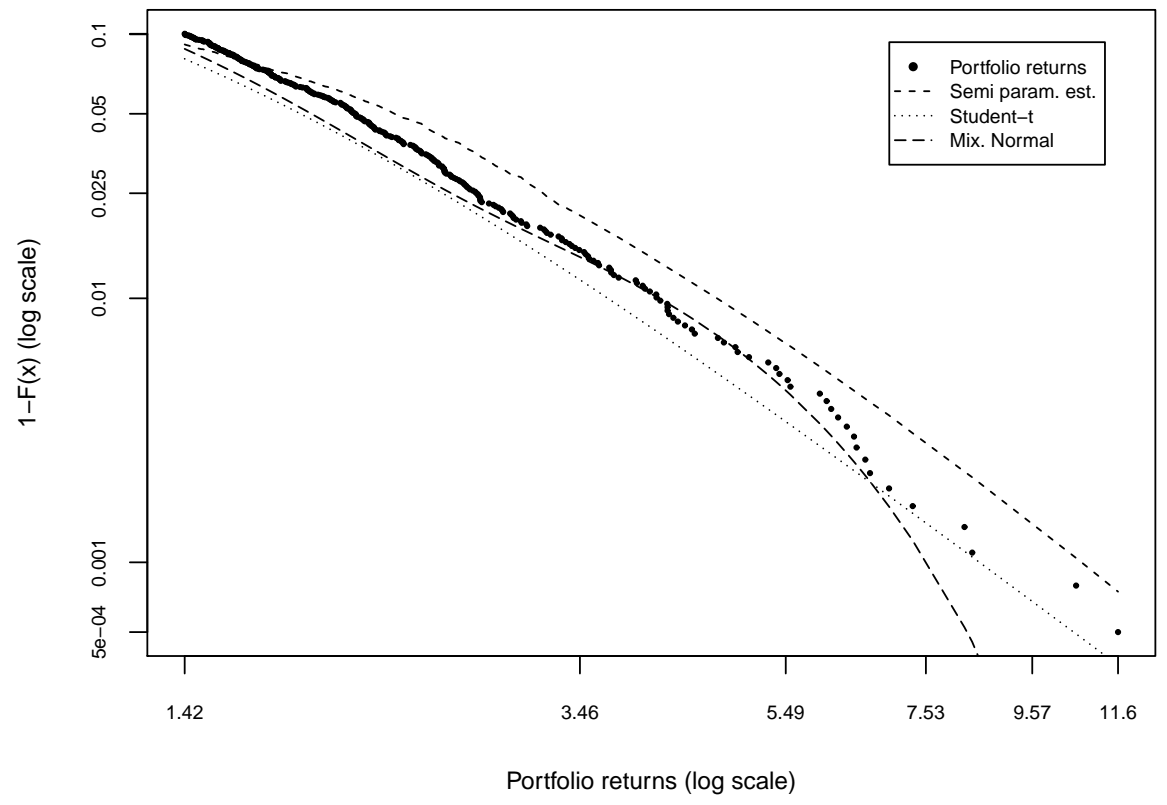

Figure 2: Observed losses (plotted in $\log$-log scale) on the fifty asset portfolio together with mixture normal, Student- $t$, and semiparametric approximation. As for the ten asset portfolio, the Student- $t$ and the mixture normal underestimate the probability of portfolio large losses.

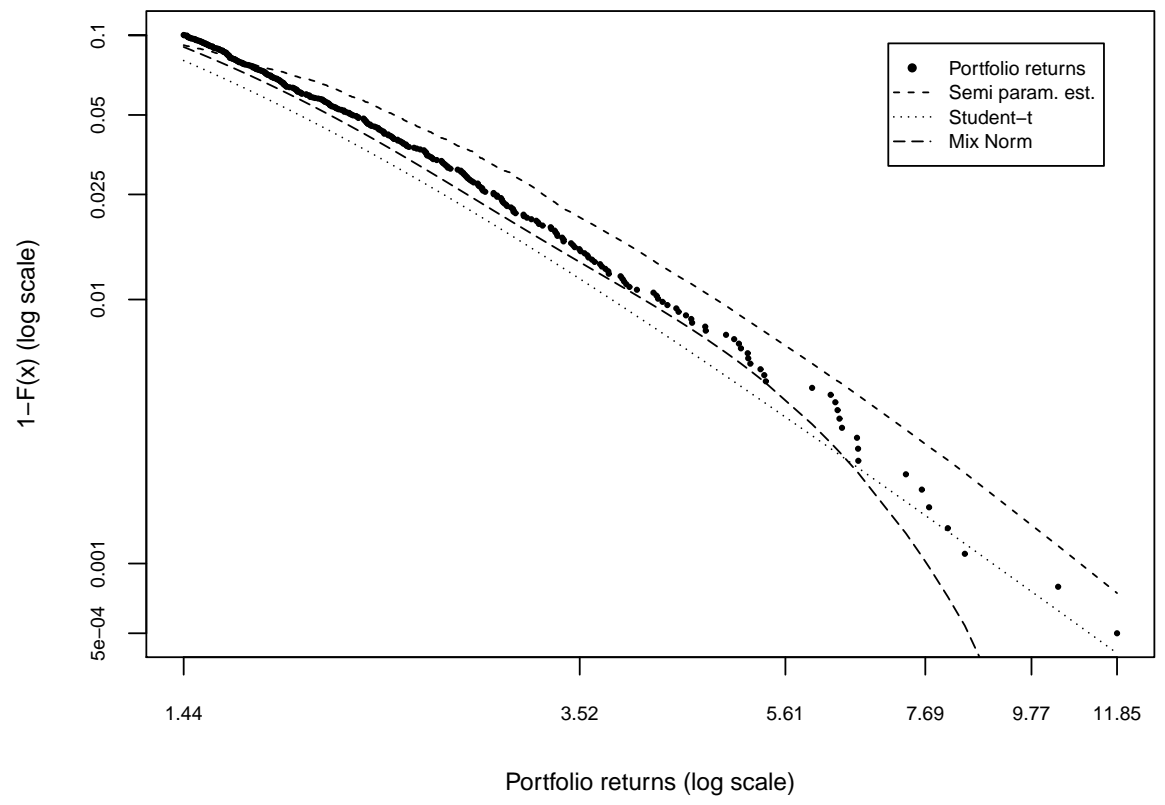

Figure 3: Observed losses (plotted in log-log scale) on the one hundred asset portfolio together with mixture normal, Student- $t$, and semiparametric approximation. Note that the semiparametric estimates do not deteriorate as the number of assets in the portfolio increases from ten to fity, and then to one hundred. 


\subsection{Semiparametric VaR estimation}

In this section we present the empirical estimates of VaR at different confidence levels and corresponding formal backtesting. We use the three portfolios with ten, fifty and one hundred components in the estimation of VaR using the Student- $t$ and the mixture of normals as benchmark models for the semiparametric estimator.

The $100 \alpha \%$ VaR is the negative of the quantile of probability $1-\alpha$ of the portfolio returns distribution. In most applications $\alpha$ varies between $95 \%$ and $99 \%$ although in certain cases, as operational risk, the Basel II Accord requires the value of 99.9\%. Formally, for a confidence level $\alpha \in(0,1)$, the $100 \alpha \%$ VaR for period $t+1$, conditional on the information available up to time $t$, is given by

$$
\operatorname{VaR}_{t+1}^{\alpha}=-\inf \left\{r \in \mathbb{R}: P\left(R_{t+1} \leq r \mid \mathcal{F}_{t}\right) \geq 1-\alpha\right\}
$$

where $R_{t}$ is the random variable representing the return in period $t$, and $\mathcal{F}_{t}$ represents the information available at time $t$.

From its definition, the $100 \alpha \%$ VaR is given by the inverse of the tail distribution estimated in the previous section at the level $1-\alpha$. The estimates obtained for the $99 \%, 97.5 \%, 95 \%$ and $90 \%$ VaR are listed in Table 3. If the estimate for the $100 \alpha \%$ VaR is accurate we would expect to observe $(1-\alpha) \%$ of return losses larger than VaR. We call the return losses larger than VaR violations. We report the percentage of violations in Table 3.

In order to test the accuracy of the estimated VaR values we use the backtesting methodology as in Christoffersen (1998). The procedure consists of three tests: unconditional coverage, independence, and conditional coverage. These are based on likelihood ratio statistics. The unconditional cover backtest tests the null hypothesis of the number of violations being equal to $1-\alpha$. Apart from being equal to the target level, it is desirable that the VaR violations are spread over time and not that large losses cluster in a small space of time. The independence backtest tests the null hypothesis of the violations being independent. The third backtest, 
conditional coverage, tests jointly the two previous hypotheses, correct number of violations and independence. We refer to Christoffersen (1998) for detailed formulae.

Because of its relevance for practice we also consider the so-called three color scheme from Basel $\mathrm{II}^{4}$. According to this criteria a VaR model is acceptable if it falls in the "green zone", it is disputable if it falls in the "yellow zone" and it is seriously flawed if it belongs to the "red zone". The color classification of a VaR model is a function of the number of violations it produces. A model is in the green zone if the number of violations is below the $95 \%$ quantile of a binomial distribution with probability of success 0.01 . The model is in the yellow zone if the number of violations is between the $95 \%$ and the $99.99 \%$ quantiles of the same binomial distribution. A model is classified in the red zone if the number of violations is above the $99.99 \%$ quantile.

Referring to the results in Table 3 we start by observing that the values of the VaR estimates for the Student- $t$ model are consistently lower than the VaR estimates obtained by the mixture normal, which in turn are lower than the estimates given by the semiparametric estimator. Given that this happens in all but one of the cases considered the results give a clear ranking of the relative levels of VaR obtained with each model.

Concerning the percentage of violations the results are striking. The semiparametric model only underestimates VaR in one case. The student- $t$ underestimates VaR in all cases and the mixture of normals underestimates VaR in nine out of twelve cases. The test for the percentage of violations, the unconditional coverage $\left(\mathrm{LR}_{u c}\right)$ test, overall also gives good results. The mixture of normals has the larger number of p-values above $10 \%$, next is the semiparametric estimator and the last is the Student- $t$ model with six out twelve p-values below $10 \%$. Although the mixture of normals performs well in the percentage of violations test we have to note that above the $99 \%$ quantile this model cannot be used given that it completely fails to model the tail for these high quantile levels (recall Figures 1, 2, and 3).

\footnotetext{
${ }^{4}$ Basel Committee on Banking Supervision (1996).
} 
According to the Basel criteria the semiparametric model is acceptable in all cases, where it always falls in the "green zone", except one where it falls in the "red zone". The mixture of normals is acceptable in all cases and the Student- $t$ model is acceptable in half of the cases and falls in the "yellow zone" in the other half of the cases.

The p-values for the independence test $\left(\mathrm{LR}_{\text {ind }}\right)$ are lower. This is expected given that these are models for the unconditional returns distribution. The semiparametric and the mixture of normals perform better than the Student- $t$ model. All the models obtain higher p-values in the independence test at higher quantiles than at lower quantiles. This indicates that clustering affects more the estimation of VaR at lower than at higher quantiles. The fact that the test has higher p-values for higher confidence levels of VaR shows that the semiparametric estimator is particularly suitable to compute probabilities of very large losses.

The conditional coverage test $\left(\mathrm{LR}_{c c}\right)$ combines the unconditional coverage and the independence tests. As a consequence, the models have higher p-values at higher confidence levels. This is a consequence of the results from the independence test.

A very important result is that, consistently across all portfolios, the VaR estimates obtained by the semiparametric model do not deteriorate significantly when the number of portfolio components increases from ten to one hundred. We can see that by comparing the percentage of violations, and the p-values for the different tests between the three portfolios. Hence, in situations where it is important to model the interaction between very high losses on different assets, the semiparametric estimator is a parsimonious effective alternative to univariate models.

\section{Conclusion}

In this study we estimate the tail risk for multi-asset portfolios. We show that using a semiparametric estimator it is possible to obtain accurate estimates of the probability of very large losses, capturing simultaneously the individual behaviour of assets and the interactions be- 
Table 3: Performance of the semiparametric tail estimator for $99 \%, 97.5 \%, 95 \%$, and $90 \%$ VaR.

\begin{tabular}{|c|c|c|c|c|c|c|}
\hline \multirow[b]{2}{*}{ Portfolio } & \multicolumn{3}{|c|}{$99 \% \mathrm{VaR}$} & \multicolumn{3}{|c|}{$97.5 \% \mathrm{VaR}$} \\
\hline & 10 assets & 50 assets & 100 assets & 10 assets & 50 assets & 100 assets \\
\hline \multicolumn{7}{|c|}{ Semiparametric } \\
\hline$\widehat{\mathrm{VaR}}$ & 4.961 & 4.432 & 4.514 & 3.352 & 2.987 & 3.042 \\
\hline$\%$ Viol. & 0.952 & 0.735 & 0.816 & 2.423 & 1.960 & 2.096 \\
\hline $\mathrm{LR}_{\mathrm{uc}}$ & 0.772 & 0.090 & 0.249 & 0.764 & 0.029 & 0.107 \\
\hline $\mathrm{LR}_{\text {ind }}$ & 0.045 & 0.015 & 0.023 & 0.025 & 0.002 & 0.001 \\
\hline $\mathrm{LR}_{\mathrm{cc}}$ & 0.128 & 0.012 & 0.039 & 0.078 & 0.001 & 0.001 \\
\hline \multicolumn{7}{|c|}{ Mixture Normal } \\
\hline$\widehat{\mathrm{VaR}}$ & 4.857 & 4.314 & 4.247 & 3.219 & 2.719 & 2.849 \\
\hline$\%$ Viol. & 1.061 & 0.762 & 0.952 & 2.777 & 2.504 & 2.514 \\
\hline $\mathrm{LR}_{\mathrm{uc}}$ & 0.709 & 0.130 & 0.772 & 0.290 & 0.985 & 0.984 \\
\hline $\mathrm{LR}_{\text {ind }}$ & 0.070 & 0.017 & 0.045 & 0.008 & 0.002 & 0.002 \\
\hline $\mathrm{LR}_{\mathrm{cc}}$ & 0.180 & 0.019 & 0.128 & 0.018 & 0.009 & 0.009 \\
\hline \multicolumn{7}{|l|}{ Student- $t$} \\
\hline$\widehat{\mathrm{VaR}}$ & 4.468 & 3.825 & 3.938 & 3.100 & 2.649 & 2.705 \\
\hline$\%$ Viol. & 1.225 & 1.143 & 1.089 & 2.913 & 2.722 & 2.994 \\
\hline $\mathrm{LR}_{\mathrm{uc}}$ & 0.185 & 0.392 & 0.592 & 0.117 & 0.394 & 0.062 \\
\hline $\mathrm{LR}_{\text {ind }}$ & 0.018 & 0.012 & 0.009 & 0.004 & 0.001 & 0.006 \\
\hline \multirow[t]{2}{*}{$\mathrm{LR}_{\mathrm{cc}}$} & 0.025 & 0.029 & 0.028 & 0.005 & 0.005 & 0.004 \\
\hline & \multicolumn{3}{|c|}{$95 \% \mathrm{VaR}$} & \multicolumn{3}{|c|}{$90 \% \mathrm{VaR}$} \\
\hline Portfolio & 10 assets & 50 assets & 100 assets & 10 assets & 50 assets & 100 assets \\
\hline \multicolumn{7}{|c|}{ Semiparametric } \\
\hline$\widehat{\mathrm{VaR}}$ & 2.379 & 2.198 & 2.211 & 1.446 & 1.431 & 1.464 \\
\hline \% Viol. & 4.982 & 4.301 & 4.492 & 12.169 & 9.855 & 9.719 \\
\hline $\mathrm{LR}_{\mathrm{uc}}$ & 0.960 & 0.046 & 0.151 & 0.000 & 0.770 & 0.569 \\
\hline $\mathrm{LR}_{\text {ind }}$ & 0.026 & 0.000 & 0.000 & 0.000 & 0.000 & 0.000 \\
\hline $\mathrm{LR}_{\mathrm{cc}}$ & 0.086 & 0.000 & 0.001 & 0.000 & 0.001 & 0.001 \\
\hline \multicolumn{7}{|c|}{ Mixture Normal } \\
\hline$\widehat{\mathrm{VaR}}$ & 2.345 & 1.977 & 2.101 & 1.626 & 1.397 & 1.440 \\
\hline$\%$ Viol. & 5.145 & 5.581 & 4.982 & 10.127 & 10.400 & 10.046 \\
\hline $\mathrm{LR}_{\mathrm{uc}}$ & 0.686 & 0.112 & 0.960 & 0.796 & 0.421 & 0.925 \\
\hline $\mathrm{LR}_{\text {ind }}$ & 0.047 & 0.000 & 0.000 & 0.000 & 0.000 & 0.000 \\
\hline $\mathrm{LR}_{\mathrm{cc}}$ & 0.128 & 0.000 & 0.001 & 0.000 & 0.001 & 0.000 \\
\hline \multicolumn{7}{|l|}{ Student- $t$} \\
\hline$\widehat{\mathrm{VaR}}$ & 2.270 & 1.936 & 1.966 & 1.558 & 1.324 & 1.338 \\
\hline \% Viol. & 5.417 & 5.799 & 5.690 & 11.135 & 11.053 & 11.216 \\
\hline $\mathrm{LR}_{\mathrm{uc}}$ & 0.251 & 0.030 & 0.060 & 0.024 & 0.036 & 0.015 \\
\hline $\mathrm{LR}_{\text {ind }}$ & 0.058 & 0.000 & 0.000 & 0.000 & 0.000 & 0.000 \\
\hline $\mathrm{LR}_{\mathrm{cc}}$ & 0.086 & 0.000 & 0.000 & 0.000 & 0.000 & 0.000 \\
\hline
\end{tabular}

Notes: The table reports the results from using the semiparametric, the mixture of normal distrutions and the Student- $t$ model to estimate the $99 \%, 97.5 \%, 95 \%$ and $90 \%$ VaR of equity portfolios composed of ten, fifty and one hundred assets with different market capitalization. $\widehat{\mathrm{VaR}}$ denotes the estimated VaR. The percentage number of violations which fall in the green, yellow and red Basel II color classification have italic, normal, and bold type face respectively. $\mathrm{LR}_{\mathrm{uc}}$ are the p-values obtained from the unconditional coverage test. $\mathrm{LR}_{\mathrm{ind}}$ and $\mathrm{LR}_{\mathrm{cc}}$ are the p-values of the independence test, and conditional coverage test respectively. The data covers the period from December 31, 1999 to January 1, 2014. 
tween them. One advantage of this estimation method is that there is no need to specify any dependence structure for the comovements of assets.

Multi-asset portfolio managers include in their portfolios assets which react differently to market conditions. This portfolio diversity implies complex portfolio return distributions, making the estimation of tail risk using univariate models difficult. In addition, univariate models are not enough in applications where it is important to model the individual contribution of each component for the portfolio characteristics. On the other hand, it is very difficult due to its complexity, to build a multivariate model for the intricate dynamics of such portfolios that can be reliably fit. The estimation of multivariate parametric models often calls for Monte Carlo simulation methods. Because the focus is in rare tail events, it is necessary to use computationally expensive techniques such as importance sampling. The semiparametric approach, as we show in this article, overcomes these problems.

In our study we estimate the tail risk at very high quantiles for equity portfolios. We obtain estimates for the distribution of the losses that are more accurate and conservative than those given by standard univariate approaches. We observe that the quality of the estimates does not worsen when the number of assets in the portfolio increases. To inspect the quality of the semiparametric estimator, we also compute VaR estimates using a mixture of normal distributions and a Student- $t$ models. The results from backtesting the values of VaR obtained are strikingly good. For portfolios with many assets the semiparametric procedure delivers very accurate VaR estimates up to very high quantiles.

An obvious extension of the application done here is to use the semiparametric estimator for computing expected-shortfall. This is an alternative measure of risk that given its additive property might progressively replace VaR. The semiparametric estimator can play an important role in smoothing this transition process.

Further applications of this estimation procedure include: (multi-name) derivatives valuation and hedging; estimation of joint credit defaults which is crucial for the valuation of credit 
derivatives; valuation of options depending on large market movements.

Our study uncovers a fact relating the risk-return dynamics with possible implications for portfolio selection. On the one hand stocks with small market capitalization typically have higher returns. On the other hand, they do not reveal higher tail risk. It could be interesting to explore this aspect of tail risk-return behaviour. The semiparametric estimator can be a very useful tool in empirical studies pursuing this direction of research. 
Appendix. Summary Statistics 
Table 4: Summary statistics of the asset returns portfolios.

\begin{tabular}{|c|c|c|c|c|c|c|}
\hline Firm & Mean & Std. Dev. & Skewness & Kurtosis & Shape $(\gamma)$ & s.e. $(\gamma)$ \\
\hline ABBOTT LABORATORIES & 0.0227 & 1.5437 & -0.3569 & 9.3655 & 0.2443 & 0.0553 \\
\hline AMERICAN EXPRESS $* *$ & 0.0192 & 2.4414 & -0.0129 & 8.8581 & 0.1827 & 0.0544 \\
\hline ARCHER-DANLS.-MIDL. $* *$ & 0.0364 & 2.0844 & -0.3138 & 9.9251 & 0.3119 & 0.0571 \\
\hline BANK OF NEW YORK MELLON * & -0.0052 & 2.5959 & -0.0930 & 16.3652 & 0.2697 & 0.0550 \\
\hline BOEING $* *$ & 0.0333 & 1.9815 & -0.2573 & 5.6232 & 0.0993 & 0.0542 \\
\hline CENTERPOINT EN. & 0.0034 & 2.5007 & -2.1758 & 124.0428 & 0.4746 & 0.0610 \\
\hline CLOROX $* *$ & 0.0198 & 1.5437 & -0.2944 & 10.6538 & 0.2952 & 0.0565 \\
\hline COMPUTER SCIS. & -0.0047 & 2.4781 & -2.5214 & 53.1690 & 0.3578 & 0.0570 \\
\hline CVS CAREMARK $* *$ & 0.0361 & 2.0050 & -1.2025 & 19.0172 & 0.3049 & 0.0562 \\
\hline DUKE ENERGY & 0.0127 & 1.6528 & -0.2182 & 11.2702 & 0.2086 & 0.0559 \\
\hline EMERSON ELECTRIC ** & 0.0245 & 1.8701 & -0.0747 & 6.9138 & 0.1693 & 0.0545 \\
\hline FAMILY DOLLAR STORES & 0.0356 & 2.2509 & 0.2322 & 7.2681 & 0.2442 & 0.0553 \\
\hline FRONTIER COMMUNICATIONS ** & -0.0266 & 2.1875 & 0.0012 & 10.7645 & 0.2900 & 0.0564 \\
\hline GOODYEAR TIRE \& RUB. * & -0.0084 & 3.3057 & -0.3493 & 4.7085 & 0.2691 & 0.0555 \\
\hline HELMERICH \& PAYNE ** & 0.0609 & 2.7504 & -0.3638 & 5.8388 & 0.2374 & 0.0558 \\
\hline INTERPUBLIC GP. & -0.0274 & 2.8322 & -0.3301 & 18.8821 & 0.3198 & 0.0559 \\
\hline INTL.PAPER & 0.0482 & 2.1650 & -0.0074 & 4.8295 & 0.3217 & 0.0562 \\
\hline JOHNSON CONTROLS ** & -0.0019 & 2.4318 & 0.0119 & 8.0199 & 0.1925 & 0.0547 \\
\hline KROGER & 0.0173 & 1.8605 & -1.2994 & 19.8008 & 0.3186 & 0.0573 \\
\hline LOWE'S COMPANIES ** & 0.0359 & 2.1865 & 0.3381 & 4.4605 & 0.1468 & 0.0536 \\
\hline MCDONALDS & 0.0208 & 1.5504 & -0.1949 & 6.1721 & 0.2416 & 0.0564 \\
\hline MURPHY OIL ** & 0.0456 & 2.2258 & -0.2650 & 6.8103 & 0.1745 & 0.0553 \\
\hline NORDSTROM & 0.0419 & 2.6821 & 0.1587 & 6.6384 & 0.1715 & 0.0544 \\
\hline OMNICOM GP. $* *$ & 0.0137 & 1.9246 & -0.3443 & 9.8132 & 0.3079 & 0.0558 \\
\hline PEPCO HOLDINGS * & -0.0067 & 1.5004 & -0.3624 & 10.3311 & 0.2734 & 0.0562 \\
\hline PITNEY-BOWES ** & -0.0191 & 1.9245 & -1.8795 & 35.4871 & 0.3480 & 0.0566 \\
\hline ROBERT HALF INTL. & 0.0303 & 2.5179 & 0.7535 & 10.4213 & 0.1908 & 0.0544 \\
\hline SOUTHWESTERN ENERGY ** & 0.1034 & 3.0349 & -0.5165 & 17.2872 & 0.2813 & 0.0563 \\
\hline TECO ENERGY & -0.0034 & 1.8381 & -1.0450 & 25.8555 & 0.3468 & 0.0581 \\
\hline THERMO FISHER SCIENTIFIC ** & 0.0578 & 1.9369 & 0.3232 & 6.4942 & 0.2352 & 0.0553 \\
\hline UNITED TECHNOLOGIES & 0.0372 & 1.8215 & -1.5880 & 33.8222 & 0.1930 & 0.0550 \\
\hline WALGREEN $* *$ & 0.0183 & 1.7550 & -0.0197 & 6.9326 & 0.1778 & 0.0545 \\
\hline WISCONSIN ENERGY * & 0.0387 & 1.1986 & -0.0403 & 4.7297 & 0.2352 & 0.0569 \\
\hline NABORS INDS. $* *$ & 0.0048 & 2.9403 & -0.3758 & 3.9721 & 0.1571 & 0.0547 \\
\hline ADVANCED MICRO DEVC. & -0.0362 & 4.0280 & -0.4161 & 7.3926 & 0.2305 & 0.0552 \\
\hline AQUA AMERICA ** & 0.0337 & 1.6804 & 0.2085 & 5.6556 & 0.0958 & 0.0548 \\
\hline ATWOOD OCEANICS * & 0.0472 & 2.7851 & -0.2462 & 6.9482 & 0.2359 & 0.0561 \\
\hline BANK OF HAWAII $* *$ & 0.0297 & 1.9856 & -0.7735 & 16.6601 & 0.2547 & 0.0561 \\
\hline BRE PROPERTIES & 0.0251 & 2.1435 & 0.0174 & 16.2649 & 0.3670 & 0.0580 \\
\hline $\mathrm{CABOT}^{* *}$ & 0.0410 & 2.3848 & -0.4048 & 9.2235 & 0.3030 & 0.0568 \\
\hline CITY NATIONAL & 0.0214 & 2.2019 & 0.1140 & 10.3376 & 0.2806 & 0.0560 \\
\hline COMMERCE BCSH. ** & 0.0236 & 1.6649 & 0.1289 & 11.7220 & 0.2503 & 0.0562 \\
\hline CON-WAY & 0.0061 & 2.5978 & -0.2120 & 6.0404 & 0.2856 & 0.0550 \\
\hline CRANE $* *$ & 0.0367 & 2.1086 & -0.3319 & 8.5065 & 0.2958 & 0.0570 \\
\hline DOMTAR & -0.0111 & 2.9189 & -0.1485 & 9.8629 & 0.3033 & 0.0565 \\
\hline ESTERLINE TECHS. ** & 0.0581 & 2.6547 & -0.2817 & 8.8841 & 0.2230 & 0.0561 \\
\hline FIRST HORIZON NATIONAL * & -0.0223 & 2.9039 & -1.0492 & 29.5786 & 0.3475 & 0.0568 \\
\hline GRACO $* *$ & 0.0560 & 1.9464 & 0.0123 & 5.3409 & 0.0717 & 0.0549 \\
\hline HAWAIIAN ELEC.INDS. & 0.0150 & 1.2879 & -0.3005 & 6.1186 & 0.2488 & 0.0566 \\
\hline HILLSHIRE BRANDS ** & 0.0051 & 1.6558 & -0.0852 & 9.7576 & 0.3222 & 0.0566 \\
\hline
\end{tabular}

Notes: Summary statistics of the daily returns on fifty of the one hundred assets considered in this study. The remaining assets are listed in Table 5. The assets had been randomly drawn from the components of the S\&P 500, S\&P 400, and S\&P 600 in equal proportions. The assets marked with ** are used in the portfolio with fifty assets and the assets marked with $*$ are used in the portfolio with ten assets. The data covers the period from December 31, 1999 to January 1, 2014. 
Table 5: Summary statistics of the asset returns portfolios.

\begin{tabular}{|c|c|c|c|c|c|c|}
\hline Firm & Mean & Std. Dev. & Skewness & Kurtosis & Shape $(\gamma)$ & s.e. $(\gamma)$ \\
\hline HUBBELL 'B' & 0.0374 & 1.8192 & 0.1353 & 6.5296 & 0.1775 & 0.0546 \\
\hline $\operatorname{ITT} * *$ & 0.0548 & 1.8188 & 0.7006 & 11.9182 & 0.1605 & 0.0549 \\
\hline LOUISIANA PACIFIC & 0.0071 & 3.5747 & -0.3982 & 7.8902 & 0.2473 & 0.0551 \\
\hline MEREDITH ** & 0.0080 & 1.8793 & 0.0333 & 8.6521 & 0.2258 & 0.0546 \\
\hline NEW YORK TIMES 'A' & -0.0258 & 2.5236 & 0.1344 & 9.5628 & 0.2325 & 0.0540 \\
\hline OWENS \& MINOR ** & 0.0492 & 1.9668 & -0.4405 & 9.7646 & 0.2880 & 0.0567 \\
\hline PNM RES. * & 0.0208 & 2.0465 & -1.2548 & 22.1534 & 0.4000 & 0.0595 \\
\hline QUESTAR ** & 0.0579 & 2.0700 & -0.0684 & 13.6832 & 0.3147 & 0.0578 \\
\hline RPM INTL. & 0.0351 & 2.0467 & -0.6402 & 8.8315 & 0.2107 & 0.0570 \\
\hline SERVICE CORP.INTL. $* *$ & 0.0247 & 3.0312 & 0.3761 & 11.5410 & 0.2411 & 0.0560 \\
\hline SPX & 0.0266 & 2.6701 & -1.3070 & 13.1028 & 0.3514 & 0.0582 \\
\hline TERADYNE $* *$ & -0.0278 & 3.5010 & -0.0523 & 4.5457 & 0.1505 & 0.0534 \\
\hline TOOTSIE ROLL & 0.0123 & 1.5602 & 0.3096 & 10.5072 & 0.1960 & 0.0542 \\
\hline UGI ** & 0.0496 & 1.4289 & 0.1567 & 6.2257 & 0.1412 & 0.0553 \\
\hline VALMONT INDS. & 0.0584 & 2.6408 & 0.1560 & 7.1372 & 0.1887 & 0.0553 \\
\hline WESTAR EN. ** & 0.0149 & 1.6495 & -1.1005 & 20.6945 & 0.3048 & 0.0581 \\
\hline TELEPHONE \& DATA SYS. & -0.0248 & 2.1820 & -0.3304 & 10.0671 & 0.2214 & 0.0547 \\
\hline NAT.PENN BANCSHARES ** & -0.0039 & 2.7234 & 0.1128 & 10.5744 & 0.2912 & 0.0566 \\
\hline AGILYSYS & 0.0027 & 3.8834 & -0.4842 & 16.8752 & 0.3774 & 0.0570 \\
\hline AMERICAN STS.WATER ** & 0.0216 & 1.8853 & -0.1808 & 3.6511 & 0.1615 & 0.0550 \\
\hline APPLIED INDL.TECHS. & 0.0512 & 2.3772 & 0.1668 & 5.3939 & 0.1368 & 0.0552 \\
\hline $\mathrm{AZZ} * *$ & 0.0823 & 2.7201 & 0.0784 & 7.6082 & 0.2340 & 0.0564 \\
\hline BRIGGS \& STRATTON * & -0.0053 & 2.2265 & -0.2511 & 4.8100 & 0.2361 & 0.0554 \\
\hline CASTLE A M \& CO. ${ }^{* *}$ & 0.0062 & 3.6689 & 0.0473 & 11.0821 & 0.2265 & 0.0554 \\
\hline CINCINNATI BELL & -0.0582 & 3.7306 & -0.4088 & 14.0249 & 0.3715 & 0.0578 \\
\hline COMTECH TELECOM. ** & 0.0362 & 3.2085 & -0.7485 & 22.4287 & 0.2608 & 0.0558 \\
\hline CUBIC & 0.0556 & 2.6070 & 0.2984 & 5.3877 & 0.2032 & 0.0552 \\
\hline DYCOM INDS. $* *$ & 0.0017 & 3.4838 & -0.8851 & 13.7465 & 0.3219 & 0.0564 \\
\hline FOREST OIL & -0.0301 & 3.1399 & -0.4906 & 8.2967 & 0.2744 & 0.0589 \\
\hline G \& K SVS.'A' ** & 0.0169 & 2.4119 & -0.8852 & 16.2248 & 0.2477 & 0.0554 \\
\hline GETTY REALTY & 0.0103 & 2.3746 & -3.9037 & 93.1506 & 0.3846 & 0.0596 \\
\hline KAMAN $* *$ & 0.0327 & 2.8467 & -0.5481 & 6.5598 & 0.1921 & 0.0563 \\
\hline LA-Z-BOY $*$ & 0.0139 & 3.6007 & -0.2298 & 16.0837 & 0.3517 & 0.0567 \\
\hline MARCUS ** & 0.0003 & 2.9534 & -0.0790 & 8.6487 & 0.2121 & 0.0548 \\
\hline MYERS INDS. & 0.0233 & 3.1000 & -0.4440 & 12.0395 & 0.2777 & 0.0565 \\
\hline NEWPORT $* *$ & 0.0176 & 4.1282 & 0.1102 & 6.2831 & 0.1781 & 0.0539 \\
\hline PARK ELECTROCHEMICAL & 0.0073 & 2.9142 & -1.5431 & 39.0104 & 0.3074 & 0.0559 \\
\hline PENN.REIT. $* *$ & 0.0030 & 3.1374 & -0.2195 & 19.7457 & 0.4097 & 0.0595 \\
\hline PINNACLE ENTM. & 0.0059 & 3.4385 & 0.4701 & 17.5174 & 0.2057 & 0.0552 \\
\hline RLI ** & 0.0480 & 1.6001 & 0.0287 & 7.5701 & 0.2143 & 0.0557 \\
\hline RYLAND GROUP & 0.0570 & 3.2025 & -0.0268 & 2.6307 & 0.1201 & 0.0536 \\
\hline SOUTHWEST GAS ** & 0.0243 & 1.5849 & -0.0092 & 5.9871 & 0.2090 & 0.0555 \\
\hline STEPAN & 0.0473 & 2.1648 & 0.2587 & 6.5180 & 0.1280 & 0.0549 \\
\hline STURM RUGER \& CO ** & 0.0572 & 2.8946 & -1.5787 & 28.0398 & 0.3006 & 0.0568 \\
\hline TEXAS INDS. & 0.0236 & 2.9616 & -0.1442 & 8.5723 & 0.3095 & 0.0558 \\
\hline UIL HDG. $* *$ & 0.0054 & 1.5305 & -0.6714 & 9.4320 & 0.2907 & 0.0565 \\
\hline UNS ENERGY & 0.0454 & 1.6387 & 1.6822 & 30.0080 & 0.2701 & 0.0596 \\
\hline WINNEBAGO INDS. ** & 0.0285 & 3.2986 & -0.0683 & 5.9392 & 0.1831 & 0.0547 \\
\hline WOLVERINE WWD. * & 0.0617 & 2.4130 & -0.2417 & 13.8351 & 0.2266 & 0.0554 \\
\hline POWELL INDUSTRIES $* *$ & 0.0601 & 3.1043 & -0.0270 & 7.3452 & 0.2543 & 0.0561 \\
\hline
\end{tabular}

Notes: Summary statistics of the daily returns on fifty of the one hundred assets considered in this study. The remaining assets are listed in Table 4. The assets had been randomly drawn from the components of the S\&P 500, S\&P 400, and S\&P 600 in equal proportions. The assets marked with ** are used in the portfolio with fifty assets and the assets marked with $*$ are used in the portfolio with ten assets. The data covers the period from December 31, 1999 to January 1, 2014. 


\section{References}

Alexander, G. J. and Baptista, A. M. (2002). Economic implications of using a mean-VaR model for portfolio selection: a comparison with mean-variance analysis. Journal of Economic Dynamics \& Control, 26:1159-1193.

Alexander, G. J. and Baptista, A. M. (2008). Active portfolio management with benchmarking: Adding a value-at-risk constraint. Journal of Economic Dynamics \&3 Control, 32:779-820.

Ang, A. and Chen, J. (2002). Asymmetric correlations of equity portfolios. Journal of Financial Economics, 63:443-494.

Arzac, E. R. and Bawa, V. S. (1977). Portfolio choice and equilibrium in capital markets with safety-first investors. Journal of Financial Economics, 4:277-288.

Banz, R. W. (1981). The relationship between return and market value of common stocks. Journal of Financial Economics, 9:3-18.

Basak, S. and Shapiro, A. (2001). Value-at-Risk-based risk management: optimal policies and asset prices. Review of Financial Studies, 14(2):371-405.

Basel Committee on Banking Supervision (1996). Supervisory framework for the use of "backtesting" in conjunction with the internal models approach to market risk capital requirements. Technical report, Available at http://www.bis.org.

Brooks, C., Clare, A. D., Dalle Molle, J. W., and Persand, G. (2005). A comparison of extreme value theory approaches for determining value at risk. Journal of Empirical Finance, 12:339352 .

Chavez-Demoulin, V., Embrechts, P., and Sardy, S. (2011). Extreme-quantile tracking for financial time series. Forthcoming in Journal of Econometrics.

Christoffersen, P. F. (1998). Evaluating interval forecasts. International Economic Review, 39:841-862.

Daul, S., Giorgi, E. D., Lindskog, F., and McNeil, A. J. (2003). Using the grouped $t$-copula. RISK, November:73-76.

de Haan, L. and de Ronde, J. (1998). Sea and wind: Multivariate extremes at work. Extremes, $1: 7-45$.

de Haan, L. and Resnick, S. (1977). Limit theory for multivariate extremes. Z. Wahrsch. Verw. Gebiete, 40:317-337.

de Haan, L. and Resnick, S. (1993). Estimating the limit distribution of multivariate extremes. Communications in Statistics. Stochastic Models, 9(2):275-309.

Dekkers, A. L. M., Einmahl, J. H. J., and de Haan, L. (1989). A moment estimator for the index of an extreme-value distribution. The Annals of Statistics, 17(4):1833-1855.

Embrechts, P., Klüppelberg, C., and Mikosch, T. (1997). Modelling Extremal Events for Insurance and Finance. Springer-Verlag, Berlin.

Gnedenko, B. V. (1943). Sur la distribution limité du terme d'une série aléatoire. Annals of Mathematics, 44:423-453.

Gourieroux, C., Laurent, J. P., and Scaillet, O. (2000). Sensitivity analysis of values at risk. Journal of Empirical Finance, 7:225-246. 
Haas, M., Mittnik, S., and Paolella, M. S. (2004). Mixed normal conditional hetroskedasticity. Journal of Financial Econometrics, 2:211-250.

Hartmann, P., Straetmans, S., and de Vries, C. G. (2001). Asset market linkages in crisis periods. Working paper, no. 71, European Central Bank, Frankfurt.

Hill, B. M. (1975). A simple general approach to inference about the tail of a distribution. The Annals of Statistics, 3(5):1163-1174.

Hyung, N. and de Vries, C. G. (2007). Portfolio selection with heavy tails. Journal of Empirical Finance, 14:383-400.

Jansen, D., Koedijk, K. J., and de Vries, C. G. (2000). Portfolio selection with limited down-side risk. Journal of Empirical Finance, 7:247-269.

Longin, F. (2000). From value at risk to stress testing: the extreme value approach. Journal of Banking \& Finance, 24:1097-1130.

Longin, F. (2005). The choice of the distribution of asset returns: How extreme value theory can help? Journal of Banking \& Finance, 29:1017-1035.

Longin, F. and Solnik, B. (2001). Extreme correlation of international equity markets. Journal of Finance, LVI(2):649-676.

McNeil, A. J. and Frey, R. (2000). Estimation of tail-related risk measures for heteroscedastic financial time series: an extreme value approach. Journal of Empirical Finance, 7:271-300.

Poon, S. H., Rockinger, M., and Tawn, J. (2004). Extreme value dependence in financial markets: Diagnostics, models, and financial implications. The Review of Financial Studies, $17: 581-610$.

Resnick, S. (2006). Heavy-Tail Phenomena: Probabilistic and Statistical Modeling. SpringerVerlag, New York.

Roy, A. D. (1952). Safety first and holding of assets. Econometrica, 20:431-449.

Susmel, R. (2001). Extreme observations and diversification in Latin American emerging markets. Journal of International Money and Finance, 20:971-986.

Tsafack, G. and Garcia, R. (2011). Dependence structure and extreme comovements in international equity and bond markets. Journal of Banking \& Finance, 35(8):1954-1970.

Zhang, Z. and Shinki, K. (2007). Extreme co-movements and extreme impacts in high frequency data in finance. Journal of Banking and Finance, 31:1399-1415. 